\title{
Lexicon Connection Priming and Ethics: As our Sense of Human Connection Decreases, so too does our Ethical Decision-Making
}

\author{
Chris Macdonald ${ }^{1 *}$ \\ ${ }^{1}$ Skylab, United Kingdom \\ *Corresponding author: Chris Macdonald: chrismacdonald@dr.com
}

\section{OPEN ACCESS}

Citation: Macdonald C. (2020) Lexicon Connection Priming and Ethics: As our Sense of Human Connection Decreases so too does our Ethical Decision-Making. Open Science Journal 5(1)

Received: 14th December 2019

Accepted: $10^{\text {th }}$ February 2020

Published: $24^{\text {th }}$ February 2020

Copyright: (c) 2020 This is an open access article under the terms of the Creative Commons Attribution License, which permits unrestricted use, distribution, and reproduction in any medium, provided the original author and source are credited.

Funding: The author(s) received no specific funding for this work

Competing Interests: The author has declared that no competing interests exist.

\begin{abstract}
:
In an era increasingly surrounded by images, it is all too easy to overlook the power of the humble word. Recent studies have shown that images depicting human disconnection can weaken our morals. This paper reveals three new experiments that demonstrate that words can replicate this phenomenon. The results of experiments 1 and 2 show that an increase of unethical decision-making can be triggered via lexicon primes that convey the idea of human disconnection. Experiment 3 suggests that while priming the idea of human disconnection can loosen our morals, priming the idea of human connection can increase ethical decision-making. The three experiments also indicate that the primes are more effective when they are indirect (such as via the use of metaphors), and when they are created to seemingly appear to come from the participants themselves (such as asking participants to list evidence for a given statement).
\end{abstract}

Keywords: Ethics, Morality, Connection, Environmental cues, Words, Priming, Ethical decision-making, Disconnecting agents, Psychology, Alienation, Sociology, Experimental psychology, Cognition, Decisionmaking, Communications, Behavioral science, Economics, Human connection 


\section{Introduction}

A pioneering series of experiments by Professor John Bargh et al revealed that lexicon priming specific themes or stereotypes can trigger assimilation. That is to say that when primed with words based on a specific topic, one can subconsciously adopt traits that are consistent with it. One of the most famous examples involves priming participants with elderly stereotypes via word games (Bargh et al, 1996). Participants who were given words such as retired, grey, rigid, and wrinkle, walked slower after completing their tasks. They seemingly adopted a trait consistent with the hidden theme: elderly stereotypes.

In another of Bargh et al's experiments, participants primed with words such as disturb, intrude, annoyingly, and bluntly, interrupted the experimenter sooner and more often (Bargh et al, 1996). Again, the participants appeared to adopt a trait consistent with the hidden theme: rudeness.

This phenomenon has since been replicated by others. In one study, it was shown that participants primed with words associated with professors achieved higher scores on a general-knowledge quiz when compared to those primed with words associated with supermodels (Dijksterhuis et al, 1998).

This paper will further explore word-induced automatic behavior using the themes of human connection and disconnection. Previous studies have shown that participants primed with the theme of human disconnection demonstrate less ethical tendencies than those primed with the theme of human connection (Macdonald, 2019; Macdonald, 2020). This has been achieved via a combination of word and image priming (Macdonald, 2019), as well as image priming by itself (Macdonald, 2020). The experiments revealed in this paper explore if this phenomenon can also be achieved via word priming without the use of images. Can words alone make us feel less connected? Can the humble word loosen our morals?

Another goal of the experiments was to decipher how to increase the potency of the primes. And as this involved exploring different priming techniques, the experiments provide convergent evidence, thus we can better understand if the triggered responses are the result of a successful prime rather than the result of any idiosyncratic effects of the primes themselves.

While previous experiments have shown that priming the idea of human disconnection can lead to a decrease of ethical decision-making when compared to those primed with human connection, it is still uncertain which prime is affecting the results. Therefore, the question remains, do human connection primes increase ethical decisions or do human disconnection primes decrease ethical decisions? Both may be the case, yet an initial ratio is yet to be proposed. To explore this aspect, Experiment 3 includes a neutrally primed control group to obtain a base level.

\section{Experiment 1}

It has previously been shown that when participants were primed with the theme of human disconnection, they were less ethical when compared to those who were primed with the theme of human connection (Macdonald, 2019; Macdonald, 2020). The priming strategies used in the aforementioned studies included a combination of word and image tasks, as well as image tasks alone. 
Experiment 1 explores if the phenomenon can be replicated when limited to word tasks.

\section{Participants}

64 US Students took part in the experiment. In an attempt to reduce variables all participants also had the following in common: aged 18-25, caucasian ethnicity, and English-only spoken at home.

\section{Procedure}

Participants were randomly assigned to one of two groups: Connection Prime $(\mathrm{CN})$, and Disconnection Prime (D) (32 participants per group). Each group was given 15 word-based tasks (such as reordering words to complete a sentence or spotting the spelling mistakes in a paragraph). The idea being that when the participants complete the tasks they will subconsciously absorb the consistent theme. All of CN's sentences conveyed the idea of human connection, for example, "With regard to our DNA, we are over $99.9 \%$ the same." All of D's sentences conveyed the idea of human disconnection, for example, "A person's $D N A$ is totally unique."

After both groups had completed their priming tasks, they were given the same set of 8 ethically relevant scenarios and were asked to indicate on a 7 point scale how likely it would be for them to engage in the behavior described $(1=$ not at all likely, $7=$ highly likely).

Therefore, the mean of the groups will be a number from 1 to 7 , and due to the nature of the questions and their framing, a lower score would be deemed as more ethical.

The scenarios and rating scale have been used in prior experiments (Piff et al, 2012; Kouchaki et al, 2013; Macdonald, 2019; Macdonald, 2020) and have been validated in several ways (Piff et al, 2012).

*A note on the survey delivery method.

A known concern with priming experiments is that the researchers conducting the experiment might be able to influence the results (Rosenthal, 1966; Conty et al, 2010; Doyen et al, 2012), even without intending to do so. To protect against this, a third-party UK organization was used to distribute all of the surveys used in the experiments. The third-party uses an automated distribution service and they were unaware of the purpose of the survey or any hypotheses attached to it.

\section{Results}

In accordance with previous findings (Macdonald, 2019; Macdonald, 2020), the data revealed that those primed with human disconnection $(\mathrm{M}=4.16, \mathrm{SD}=$ $2.35, \mathrm{~N}=32$ ) were less ethical when compared to those who were primed with human connection $(\mathrm{M}=4.13, \mathrm{SD}=2.24, \mathrm{~N}=32)$. This further supports the hypothesis that a decreased sense of human connection can have a negative impact upon ethical decisions. It also indicates that this phenomenon can be triggered via lexicon primes alone. However, the difference between the two 
groups, although consistent with the hypothesis, was not statistically significant $(\mathrm{p}=.9)$.

The marginal difference between the prime groups could be due to the word primes being less subtle than the image primes, and therefore the primed theme might be more conscious which may be resulting in some participants attempting to consciously resist the prime. Triggering specific states of mind without full participant awareness has been key in previous priming experiments (Schweder et al, 1984; Triandis, 1989; Bargh et al, 2001). To facilitate this, Experiment 2 explores the use of fewer word tasks with less direct priming strategies. The hypothesis being that this alteration could ameliorate the subconscious absorption of the lexicon primes, and thus increase the impact upon ethical decision-making.

\section{Experiment 2}

While Experiment 1 was technically consistent with the hypothesis - that priming themes of human disconnection lowers ethical decision-making - the results were not conclusive $(\mathrm{t}[56]=-0.12, \mathrm{p}=.9)$. As a possible explanation for this is the quality of the primes, Experiment 2 uses alternative priming strategies.

\section{Participants.}

68 US Students took part in the experiment. In an attempt to reduce variables, all participants also had the following in common: aged 18-25, caucasian ethnicity, and English-only spoken at home.

\section{Procedure}

As with Experiment 1, the participants were randomly assigned to one of two groups: Connection Prime (CN), and Disconnection Prime (D) (34 participants per group). Each participant was given a set of 10 word-based tasks. 5 of the tasks were neutral and consistent across the groups such as "How many times does the letter $t$ appear in this sentence?" The remaining 5 tasks were groupspecific: connection primes for $\mathrm{CN}$ and disconnection primes for $\mathrm{D}$. This was done to reduce variables as well as rendering the primes less direct. The primes themselves also assisted with this. Rather than directly referencing the theme, the primes focus on a seemingly abstract task that metaphorically implies the theme. Here is an example priming task used for $\mathrm{CN}$ :

"Laura, Peter, David, Ben, and Susan are all holding the same piece of string as John, Helen, Stephen, and Samantha. How many people are connected by the string?"

And here is an example priming task used for D:

"Laura, Peter, David, Ben, Susan, John, Helen, Stephen, and Samantha all have their own unique detached house. Although they are completely disconnected from one another, if they were to live in an apartment block, each with their own self-contained private floor, how many floors would the apartment block need?" 
Both tasks contained the same names to reduce variables.

After completing the priming tasks, the participants were asked to complete the same set of ethically relevant questions as used in Experiment 1.

\section{Results}

In accordance with previous findings (Macdonald, 2019; Macdonald, 2020), and Experiment 1, the data revealed that those primed with the theme of human disconnection $(\mathrm{M}=4.26, \mathrm{SD}=2.33, \mathrm{~N}=34$ ) were less ethical when compared to those primed with the theme of human connection $(\mathrm{M}=4.10, \mathrm{SD}=2.33, \mathrm{~N}=$ 34). The difference between the groups is now consistent with the results of the previous connection experiments that used image primes alone (Macdonald, 2020).

As the difference between the two groups was more significant in Experiment $2(\mathrm{t}[65]=-0.73, \mathrm{p}=.46)$, the results support the hypothesis that priming less directly increases priming absorption.

\section{Experiment 3}

The final experiment explores the following question, would the potency of the effect increase even further if the prime appeared to come from the participants themselves? For example, rather than the priming strategy being somewhat passive (such as spotting typos, counting elements, or reordering words), what if the participants were to create the initial sentences themselves? One might assume that generating their own sentences about the theme may result in the participants negotiating some form of justification or confirmation, and as a result, perhaps this might facilitate greater prime accessibility.

Although there is now mounting evidence that the thought of human disconnection appears to be related to unethical decision-making, the phenomenon's mechanistic ratio is yet to be explored. In other words, do thoughts of human disconnection decrease ethical decision-making? And/or do thoughts of human connection increase ethical decision-making? If both are the case, is one significantly more potent than the other?

To explore the new set of questions effectively, Experiment 3 uses new priming tasks and includes a control group to establish a base level for ethical judgement.

\section{Participants.}

87 US Students took part in the experiment. In an attempt to reduce variables all participants also had the following in common: aged 18-25, caucasian ethnicity, and English-only spoken at home. 


\section{Procedure.}

The 87 participants were divided into three groups, Connection Prime (CN), Control Prime (C), and Disconnection Prime (D) (29 participants per group). Each group was given a task where they had to create 5 sentences relevant to their prime:

$\mathrm{CN}$ : "Please list 5 ways that humans are completely connected."

C: $\quad$ "Please write 5 random sentences that begin with the letter A."

D: "Please list 5 ways that humans are completely disconnected."

After completing the priming task, the participants were asked to complete the same set of ethically relevant questions as used in experiments 1 and 2 .

\section{Results}

Consistent with previous findings, those primed with the theme of human disconnection $(\mathrm{M}=4.46, \mathrm{SD}=2.24, \mathrm{~N}=29)$ were less ethical than those primed with the theme of human connection $(\mathrm{M}=4.00, \mathrm{SD}=2.24, \mathrm{~N}=29)$. The difference between the two groups was more significant $(\mathrm{t}[54]=-1.94, \mathrm{p}=.05$ ) than the previous experiments which supports the idea that prime potency increases when the prime appears to come from the participants themselves. Perhaps particularly, as the questions were framed in a way in which the participants had to support the prime.

The mean score of the control group-those who received neutral primes-was in between the two other groups $(\mathrm{M}=4.27, \mathrm{SD}=2.38, \mathrm{~N}=29)$. Therefore, the results suggest that while thoughts of human disconnection can decrease ethical decision-making, thoughts of human connection can increase ethical decisionmaking. As D's results were closer to C's, it suggests that thoughts of human connection are a more potent prime when it comes to the influence upon ethical decision-making (at a ratio of $1.42: 1$ ).

\section{Concluding Remarks}

This paper offers further support for a current hypothesis: that thoughts of human disconnection can trigger a decrease in ethical decision-making. It also suggests that the reverse may also be true: that thoughts of human connection can trigger an increase in ethical decision-making. And perhaps to a greater extent (1.42:1).

The varied experiment techniques shed light on some of the nuances of this phenomenon, such as the implications of the format, length, subtlety, and appeared origin of the primes.

While there is a lot of work to be done in this field, the results of the experiments reported here can remind us of a pertinent truth: In a time of widespread polarizing and decisive rhetoric, it is incredibly important to remember the power of words and the dangers of feeling less connected to one another. 


\section{References:}

Bargh J A, Chen M, Burrows L. 1996. Automaticity of social behavior: Direct effects of trait construct and stereotype activation on action. Journal of Personality and Social Psychology 71(2) 230-244

Bargh JA, Gollwitzer P, Lee-Chai A, Barndollar K, Trötschel R. 2001. The automated will: Nonconscious activation and pursuit of behavioral goals. Journal of Personality and Social Psychology 81: 1014-1027.

Conty L, Gimmig D, Belletier C, George N, Huguet P. 2010. The cost of being watched: Stroop interference increases under concomitant eye contact. Cognition 115: 133-139

Dijksterhuis A, Spears R, Postmes T, Stapel D, Koomen W, Knippenberg A, Scheepers D. 1998. Seeing one thing and doing another: Contrast effects in automatic behavior. Journal of Personality and Social Psychology 75(4) 862-871

Doyen S, Klein O, Pichon CL, Cleeremans A. 2012. Behavioral Priming: It's All in the Mind, but Whose Mind? PLoS ONE 7(1) e29081

Macdonald C. 2019. Money, a disconnecting agent: Reminders of money trigger a feeling of disconnection which increases the likelihood of unethical decisions. Open Science Journal, 4:(1) 110

Macdonald C. 2020. Graphical connection priming and ethics: As our sense of human connection decreases, so too does our ethical decision-making. Open Science Journal, 5:(1) 1-6

Rosenthal R. 1966. Experimenter effects in behavioral research. New York: Appleton-Century-Crofts

Schweder RA, Bourne L. 1984. Does the concept of the person vary cross-culturally? In Schweder RA, Levine RA (Eds.), Culture theory: essays on mind, self, and emotion. New York: Cambridge University Press 158-199

Triandis HC. 1989. The self and social behavior in differing cultural contexts. Psychological Review 96: $506-520$ 